\title{
A Novel Four-Wave Mixing Compensator
}

\author{
J. Y. Huh, S. B. Jun, J. H. Lee, and Y. C. Chung, Fellow, IEEE
}

\begin{abstract}
We propose and demonstrate a novel four-wavemixing (FWM) compensator made of a highly nonlinear fiber and a pump laser. This technique utilizes the additional $\pi / 2$ phase delay occurring in every FWM process. Thus, the FWM components generated in the transmission fiber can be cancelled out by the FWM components generated within the FWM compensator. We have experimentally confirmed that the proposed technique could effectively suppress the FWM crosstalk generated in the transmission fiber and improve the system's performance.
\end{abstract}

Index Terms-Compensation, four-wave mixing (FWM), highly nonlinear fiber (HNLF).

\section{INTRODUCTION}

$\mathbf{F}$ OUR-WAVE mixing (FWM) is one of the most crucial limiting factors for the high-capacity wavelengthdivision-multiplexing (WDM) system having densely spaced channels. Previously, there have been several attempts to overcome this limitation by placing an optical phase conjugation (OPC) module in the middle of the transmission link [1]-[3]. However, this method requires symmetrical distribution of the optical power and fiber dispersion before and after the OPC module, which is extremely difficult to realize in practice. In this letter, we propose and demonstrate a novel FWM compensator made of a highly nonlinear fiber (HNLF) and a pump laser. The configuration of the proposed compensator is similar to typical fiber-based OPC modules. However, unlike the OPC module, the proposed FWM compensator is used right in front of the receiver. This compensator converts the wavelengths of the signals and the FWM components (generated in the transmission fiber) to the opposite side of the wavelength region with respect to the pump wavelength. In addition, numerous FWM components are newly generated within this FWM compensator. However, these newly generated FWM componnets are out of phase with the components generated in the transmission fiber due to the additional $\pi / 2$ phase delay occurred in every FWM process [4]. As a result, the FWM components generated in the transmission fiber could be cancelled out by the FWM components generated in the proposed compensator. We confirmed that this technique could effectively compensate the FWM crosstalk generated in the transmission fiber and improve the bit-error rate (BER) of WDM signals.

Manuscript received August 25, 2006; revised November 1, 2006.

The authors are with the Korea Advanced Institute of Science and Technology, Department of Electrical Engineering, Daejeon 305-701, Korea (e-mail: ychung@ee.kaist.ac.kr).

Color versions of one or more of the figures in this paper are available online at http://ieeexplore.ieee.org.

Digital Object Identifier 10.1109/LPT.2006.888994
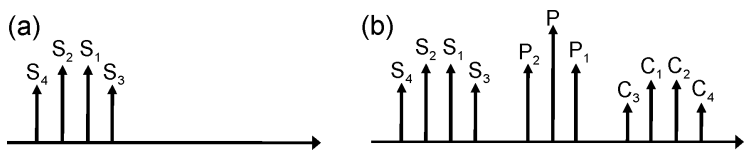

Fig. 1. Optical spectra at the (a) input and (b) output of the proposed FWM compensator.

\section{PRINCIPLE OF OPERATION}

The electrical field of the FWM component generated in optical fiber can be described as [4]

$$
E_{1}(L)=i \frac{2 \pi \omega_{1}}{n_{1}}\left(D \chi_{1111}\right) E_{2}^{*} E_{3} E_{4} \times \exp \left(-\frac{1}{2} \alpha L\right) L_{\mathrm{eff}}
$$

where $n_{1}, D, \chi_{1111}, \alpha, L$, and $L_{\text {eff }}$ are the fiber-core refractive index, degeneracy factor, electric susceptibility, fiber attenuation coefficient, fiber length, and effective fiber length, respectively. Using this equation, the optical frequency and phase of the FWM component can be obtained as $\omega_{1}=-\omega_{2}+\omega_{3}+\omega_{4}$ and $\phi_{1}=-\phi_{2}+\phi_{3}+\phi_{4}-\pi / 2$, respectively. This relation indicates that the FWM process causes an additional phase delay of $\pi / 2$. We utilized this additional phase delay for the FWM compensation.

To describe the operating principle of the proposed FWM compensation technique, we assume that two WDM signals are transmitted through the transmission fiber. Fig. 1(a) shows the FWM components $\left(S_{3}\right.$ and $S_{4}$ ) generated at both sides of WDM signals $\left(S_{1}\right.$ and $\left.S_{2}\right)$ after the transmission. These signals and FWM components $\left(S_{1}, S_{2}, S_{3}\right.$, and $\left.S_{4}\right)$ are sent to the proposed FWM compensator made of an HNLF and a pump laser. For the maximum FWM efficiency, it is necessary to operate the pump laser slightly offset from the zero-dispersion wavelength $\left(\lambda_{o}\right)$ of the HNLF due to the Kerr phase-shift effect [5]. However, we operate the pump laser precisely at the $\lambda_{o}$ of the HNLF to maximize the flat FWM bandwidth [6]. In the FWM compensator, the wavelengths of $S_{1}, S_{2}, S_{3}$, and $S_{4}$ are converted to the opposite side with respect to the pump wavelength. In addition, numerous FWM components (including $P_{1}$ and $P_{2}$ ) are newly generated, as shown Fig. 1(b). Thus, the spectral components, $C_{1}, C_{2}, C_{3}$, and $C_{4}$, are comprised of the wavelength-converted components of $S_{1}, S_{2}, S_{3}$, and $S_{4}$, and the newly generated FWM components at their corresponding wavelengths within the HNLF, respectively. For example, $C_{3}$ can be considered as a sum of the wavelength-converted component of $S_{3}$ and the newly generated FWM components in the HNLF such as $P P_{2} S_{1}^{*}$. Thus, $C_{3}$ can be described as

$$
\begin{aligned}
C_{3} & =\eta_{1} P^{2} S_{3}^{*}+\eta_{2} P P_{2} S_{1}^{*}+\cdots \\
& =\eta_{1} P^{2} S_{3}^{*}+\eta_{2} P\left(\eta_{3} P S_{2} S_{1}^{*}\right) S_{1}^{*}+\cdots
\end{aligned}
$$




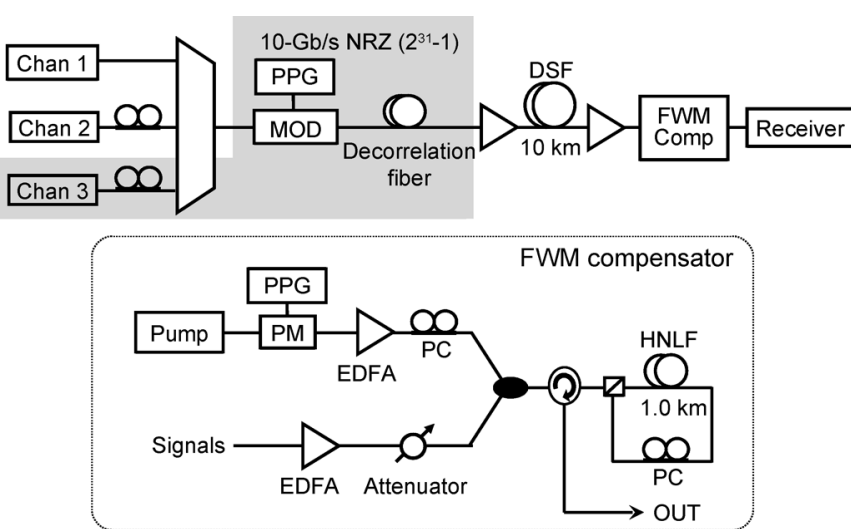

Fig. 2. Experimental setup (PPG: pulse pattern generator. MOD: intensity modulator. PM: phase modulator. PC: polarization controller. EDFA: erbium-doped fiber amplifier).

where $\eta_{1}, \eta_{2}$, and $\eta_{3}$ are the FWM coefficients of their corresponding FWM processes, respectively. We assume that the chromatic dispersion of the transmission fiber is small to ignore the differences in these FWM coefficients. If the FWM efficiency in HNLF is not too large, we can consider only the first two terms in (2) and neglect the other terms. However, the phases of the first and second terms are determined by

$$
\begin{aligned}
\phi_{\text {first }} & =2 \phi_{p}-\left(2 \phi_{1}-\phi_{2}-\frac{\pi}{2}\right)-\frac{\pi}{2} \\
& =2 \phi_{p}-2 \phi_{1}+\phi_{2} \\
\phi_{\text {second }} & =\phi_{p}+\left(\phi_{p}+\phi_{2}-\phi_{1}-\frac{\pi}{2}\right)-\phi_{1}-\frac{\pi}{2} \\
& =2 \phi_{p}-2 \phi_{1}+\phi_{2}-\pi
\end{aligned}
$$

since $\phi_{3}=2 \phi_{1}-\phi_{2}-\pi / 2$ [4]. These relations clearly show that the first and second terms in (2) are out of phase. Thus, we should be able to suppress $C_{3}$ by properly adjusting the signal powers $\left(S_{1}, S_{2}, S_{3}\right.$, and $\left.S_{4}\right)$ and the pump power $(P)$ incident on the FWM compensator.

\section{EXPERIMENTS AND RESULTS}

To demonstrate the feasibility of the proposed FWM compensator, we first performed a two-channel experiment by using the setup shown in Fig. 2 (except the shaded area). We transmitted two WDM signals separated by $100 \mathrm{GHz}$ through the $10-\mathrm{km}$ long dispersion-shifted fiber (DSF). We used a short DSF to avoid the problems associated with chromatic dispersion. The wavelengths of WDM signals were 1547.72 and $1548.51 \mathrm{~nm}$, while $\lambda_{0}$ of the DSF was $1548.10 \mathrm{~nm}$. We increased the signal powers incident on the DSF up to $8 \mathrm{dBm}$ and adjusted the polarization controller to maximize the FWM crosstalk. The inset in Fig. 2 shows the proposed FWM compensator implemented by using a 1-km-long HNLF and a pump laser. The signal power incident on the HNLF was set to be $4 \mathrm{dBm} /$ channel. For the polarization-independent operation, a polarization beam splitter was used at the input of the HNLF installed in a loop configuration [6]. We operated the pump laser precisely at the $\lambda_{0}$ of HNLF (i.e., $1553.25 \mathrm{~nm}$ ) to maximize the bandwidth of the proposed FWM compensator. In addition, to suppress the stimulated Brillouin scattering (SBS), the pump laser was modulated

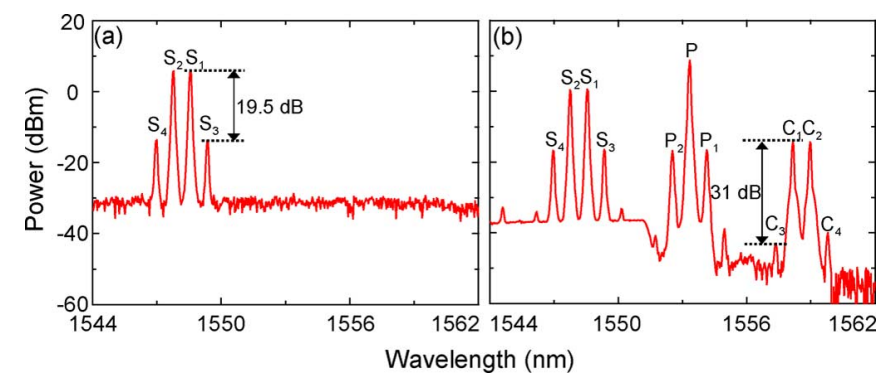

Fig. 3. Measured optical spectra at the (a) input and (b) output of the FWM compensator.

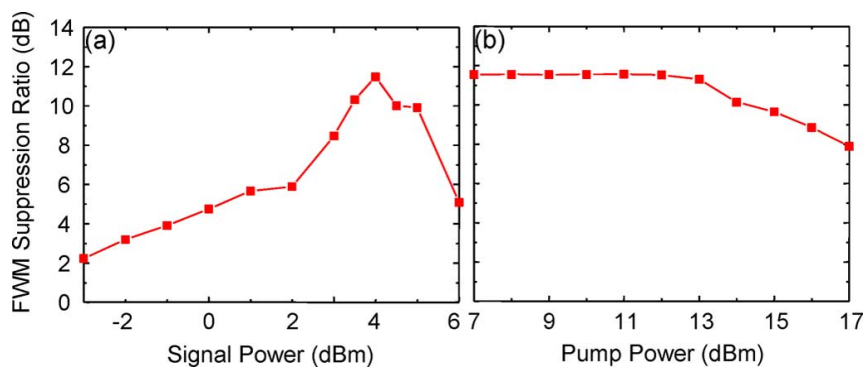

Fig. 4. (a) FWM suppression ratio measured as a function of signal power incident on HNLF. (b) FWM suppression ratio measured as a function of pump power incident on HNLF.

with 3-Gb/s signal (pattern length $=2^{31}-1$ ) by using an external phase modulator. The pump power incident on the HNLF was $12 \mathrm{dBm}$. Fig. 3(a) and (b) shows the optical spectra measured at the input and output of the FWM compensator, respectively. From these figures, we concluded that the proposed compensator improved the signal-to-FWM crosstalk ratio from 19.5 to $31 \mathrm{~dB}$ (i.e., by $11.5 \mathrm{~dB}$ ). We also measured the FWM suppression ratios while varying the signal and pump powers. Fig. 4(a) shows that this ratio is quite sensitive to the signal powers incident on the FWM compensator. This was due to the fact that the second term in (2) was more sensitive to the signal powers incident on the HNLF than the first term (since the second term involved in two different FWM processes while the first term was generated by only one process). On the other hand, Fig. 4(b) shows that the performance of the proposed FWM compensator is not sensitive to the pump power. This was because the pump power affected the magnitudes of the first and second terms in (2) in the same way. However, when the pump power was increased to be higher than $13 \mathrm{dBm}$, the performance of the FWM compensator began to be deteriorated as the other terms in (2) became too large to be neglected. Thus, for the precise FWM compensation, it would be necessary to properly control the signal powers incident on the HNLF and limit the pump power (to the value determined by the characteristics of HNLF).

We evaluated the performance of the proposed FWM compensator by using three modulated WDM signals (i.e., including the shaded area in Fig. 2). The additional laser operated at $1549.32 \mathrm{~nm}$. We modulated these lasers, spaced at $100 \mathrm{GHz}$, with $10-\mathrm{Gb} / \mathrm{s}$ NRZ signal (pattern length: $2^{31}-1$ ) by using an external intensity modulator. These modulated signals were decorrelated and then sent to $10-\mathrm{km}$-long DSF. After transmission, we measured the eye diagrams and receiver 


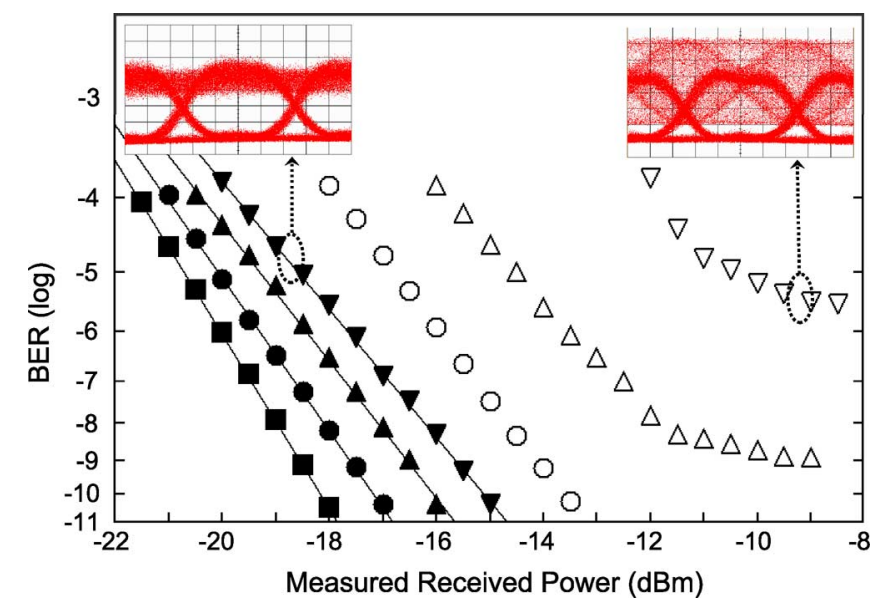

Fig. 5. BER curves measured with and without using the proposed FWM compensators. (The open symbols $(0, \triangle, \nabla)$ indicate the BER curves measured without using the FWM compensator. The closed symbols $(\bullet, \boldsymbol{\Delta}, \boldsymbol{\nabla})$ indicate the BER curves measured with using the FWM compensator. The circles $(0$, $\bullet)$, triangles $(\triangle, \boldsymbol{\Delta})$, and reverse triangles $(\nabla, \boldsymbol{\nabla})$ indicate the cases when the signal powers incident on the 10-km-long DSF link were set to be 5.5, 6.0, and $6.5 \mathrm{dBm} /$ channel, respectively. The closed square $(\mathbf{\square})$ is the baseline measured in a back-to-back configuration without using the FWM compensator.

sensitivities of the WDM signals with and without using the FWM compensator. Fig. 5 shows the measured BER curves of the center channel. When we set the signal powers incident on the transmission fiber to be $5.5 \mathrm{dBm} / \mathrm{channel}$, the power penalty induced by FWM on the central channel was measured to be $4.5 \mathrm{~dB}\left(\right.$ at $\left.\mathrm{BER}=10^{-9}\right)$. In addition, as we increased the signal powers to be $>6 \mathrm{dBm} /$ channel, error-free transmission was no longer possible due to the BER floor. However, we could overcome this limitation by using the proposed FWM compensator. For example, the power penalties were reduced to 1.0, 2.1 , and $3.0 \mathrm{~dB}$ when we set the signal powers incident on the transmission fiber to be 5.5, 6.0, and $6.5 \mathrm{dBm}$, respectively. The larger power penalties were observed at higher signal powers due to the limitations of the FWM compensator imposed by the characteristics of the HNLF used in this experiment. The insets in Fig. 5 show the eye diagrams measured before and after FWM compensator when the signal power incident on the transmission fiber was set to be $6.5 \mathrm{dBm}$. The eye opening was substantially improved after passing through the proposed FWM compensator. The compensated eye diagram had a relatively thick mark level as we phase-modulated the pump laser to suppress the effect of SBS.

\section{SUMMARY}

We have proposed and demonstrated a novel FWM compensator implemented by using HNLF and a pump laser. In the proposed technique, the FWM component generated in the transmission fiber was compensated by the FWM component generated in the FWM compensator. This was because these FWM components were out of phase from each other due to the additional $\pi / 2$ phase delay occurring in every FWM process. We experimentally confirmed that the proposed compensator could suppress the FWM crosstalk by as much as $11.5 \mathrm{~dB}$. We also confirmed that the proposed compensator could substantially improve the quality of the signal corrupted by the FWM crosstalk. For example, when we transmitted three $10-\mathrm{Gb} / \mathrm{s}$ signals (launched power: $5.5 \mathrm{dBm} /$ channel, channel spacing: $100 \mathrm{GHz}$ ) into a 10-km-long DSF link, the FWM-induced power penalty of the center channel was measured to be $4.5 \mathrm{~dB}$ (at BER $=10^{-9}$ ). However, we could reduce this penalty to $1 \mathrm{~dB}$ by using the proposed FWM compensator.

\section{REFERENCES}

[1] S. Watanabe and T. Chikama, "Cancellation of four-wave mixing in multichannel fibre transmission by midway optical phase conjugation," Electron. Lett., vol. 30, no. 14, pp. 1156-1157, Jul. 1994.

[2] S. Watanabe, "Cancellation of four-wave mixing in a single-mode fiber by midway optical phase conjugation," Opt. Lett., vol. 19, no. 17, pp. 1308-1310, Sep. 1994.

[3] K. Kikuchi, "Elimination of four-wave mixing in dispersion-shifted optical fibers by using midway optical phase conjugation in a semiconductor optical amplifier," in Tech. Dig. CLEO'96, Jun. 1996, pp. 42-43.

[4] K. O. Hill, D. C. Johnson, B. S. Kawasaki, and R. I. MacDonald, "CW three-wave mixing in single-mode optical fibers," J. Appl. Phys., vol. 49, no. 10, pp. 5098-5106, Oct. 1978.

[5] G. P. Agrawal, Nonlinear Fiber Optics, 3rd ed. New York: Academic, 2001, ch. 10

[6] S. Watanabe, S. Takeda, and T. Chikama, "Interband wavelength conversion of $320 \mathrm{~Gb} / \mathrm{s}(32 \times 10 \mathrm{~Gb} / \mathrm{s})$ WDM signal using a polarization-insensitive fiber four-wave mixer," in Tech. Dig. ECOC'98, Sep. 1998, pp. 83-87. 